\title{
Experimental and Computational Study on Flapping Wings with Bio-Inspired Hover Kinematics
}

\author{
Ruben B. R. Vandenheede* \\ Delft University of Technology, 2600 AA Delft, Netherlands \\ Luis P. Bernal,, \pm Christian L. Morrison,,$\underline{ \pm}$ Abhijit Gogulapati, $\underline{\underline{s}}$ \\ Peretz P. Friedmann, $\mathbb{1}$ and Chang-Kwon Kang-* \\ University of Michigan, Ann Arbor, Michigan 48109 \\ and \\ Wei Shyy辛 \\ Hong Kong University of Science and Technology, Kowloon, Hong Kong, \\ People's Republic of China

\section{DOI: $10.2514 / 1 . J 052644$}

\begin{abstract}
In this paper, force and particle-image-velocimetry vorticity measurements of biologically inspired hover kinematics are compared to corresponding results of an unsteady aerodynamic vortex model and a Navier-Stokes (NS) solver. The Reynolds number and the reduced frequency are $4.8 \times 10^{3}$ and 0.38 , respectively. Three kinematics derived from the measured hovering kinematics of an Agrius convolvuli are considered: 1) without elevation angle, 2) elevation angle accounted in the pitch angle, and 3) pure sinusoidal pitch-plunge neglecting higher harmonics. The Navier-Stokes computations show good qualitative agreement with experiments with consistent underprediction. The time-averaged thrust coefficients obtained using Navier-Stokes computations are 82 to $87 \%$ of the corresponding force measurements. The standard deviation of time history of thrust coefficients, also normalized by the measured time-averaged values, is 13 to $20 \%$. The underprediction is possibly due to blockage effects in the experiments, also reflected in lower values of the vorticity compared to particle-image-velocimetry measurements. The unsteady aerodynamic vortex model captures some of the peaks in a qualitative manner. The relative difference in the timeaveraged forces and standard deviation are 8 to $18 \%$ and 66 to $93 \%$, respectively. The differences in prediction of time histories are not reflected in the estimation of time-averaged forces due to cancellation effects, wherein the forces are underpredicted in the first half of the stroke and overpredicted in the second half. The discrepancies are attributed to the simplifying assumptions in the unsteady aerodynamic vortex model, which overpredicts the vorticity in the leading-edge vortex and results in significant differences in the wing-wake interaction process.
\end{abstract}

\section{Nomenclature}

$\begin{array}{ll}A & =\text { area swept by the wing, } \mathrm{m}^{2} \\ C_{L} & =\text { lateral force coefficient } \\ C_{T} & =\text { thrust coefficient }\end{array}$

Presented as Paper 2013-0066 at the 51st Aerospace Sciences Meeting Including the New Horizons Forum and Aerospace Exposition, Grapevine, TX, 7-10 January 2013; received 25 February 2013; revision received 22 July 2013; accepted for publication 30 July 2013; published online 28 February 2014. Copyright $\odot 2013$ by Ruben Vandenheede, Luis Bernal, Christian Lee Morrison, Chang-Kwon Kang, Wei Syy, Abhijit. Published by the American Institute of Aeronautics and Astronautics, Inc., with permission. Copies of this paper may be made for personal or internal use, on condition that the copier pay the $\$ 10.00$ per-copy fee to the Copyright Clearance Center, Inc., 222 Rosewood Drive, Danvers, MA 01923; include the code 1533-385X/14 and $\$ 10.00$ in correspondence with the CCC.

*Graduate Research Assistant, Department of Aerospace Engineering; currently Gasflow Architect, ASML Veldhoven; vdh.ruben@gmail.com.

${ }^{\dagger}$ Associate Professor, Department of Aerospace Engineering; 1pb@umich .edu.

${ }^{\ddagger}$ Graduate Research Assistant, Department of Aerospace Engineering; cmorrisz@umich.edu.

${ }^{\S}$ Post-Doctoral Research Fellow, Department of Aerospace Engineering; currently Department of Mechanical and Aerospace Engineering, The Ohio State University; Abhijitg@umich.edu.

"Francois-Xavier Bagnoud Professor, Department of Aerospace Engineering; peretzf@umich.edu. Fellow AIAA.

**Post-Doctoral Research Fellow, Department of Aerospace Engineering; currently Assistant Professor, Department of Mechanical and Aerospace Engineering, University of Alabama in Huntsville; Chang-kwon.Kang@uah edu. Member AIAA.

${ }^{\dagger}$ Provost \& Chair Professor, Department of Mechanical and Aerospace Engineering, Hong Kong University of Science and Technology; currently Adjunct Professor, Department of Aerospace Engineering, University of Michigan; weishyy@ust.hk. Fellow AIAA.

$\begin{array}{lll}c & =\text { mean chord length, } \mathrm{m} \\ F_{\text {lateral }} & =\text { lateral force, } \mathrm{N} \\ f & =\text { flapping frequency, } \mathrm{Hz} \\ h & =\text { plunge position experiment } \\ h_{0} & =\text { plunge amplitude experiment } \\ k & =\text { reduced frequency } \\ L_{\text {ref }} & =\text { reference length } \\ M_{x} & =\text { moment around the } x \text { axis, } \mathrm{N} \cdot \mathrm{m} \\ P & =\text { power, } \mathrm{W} \\ R & =\text { semispan, } \mathrm{m} \\ R e & =\text { Reynolds number } \\ T & =\text { thrust force, } \mathrm{N} \\ U_{\text {ref }} & =\text { reference velocity, } \mathrm{m} / \mathrm{s} \\ \alpha & =\text { feathering angle, rad } \\ \delta & =\text { pitch angle, rad } \\ \theta & =\text { elevation angle } \\ \nu_{a} & =\text { viscosity of air } \\ \nu_{w} & =\text { viscosity of water } \\ \rho & =\text { density } \\ \Phi & =\text { flapping amplitude, rad } \\ \phi & =\text { flapping angle } \\ \cdot & =\text { first time derivative } \\ - & =\text { averaged quantity }\end{array}$

\section{Introduction}

$\mathbf{F}$ LAPPING wings have unique characteristics that can be exploited in the development of micro aerial vehicles; for example, flapping wings perform very well in small spaces, they are very small, and they have low energy consumption. Applications can range from reconnaissance in confined spaces to search and rescue in damaged buildings. Of particular interest is the aerodynamics of small birds and insects in hover, which demonstrate the agility 
necessary for those missions. A well-studied example of a biological flapping flyer is the hawkmoth. This moth is an excellent study object for a number of reasons [1]. The wing beat of the moths is strikingly consistent. This consistent wing beat holds for an individual moth as well as comparing different moths. The main features dominant in insect flapping flight are present in the stroke of the hawkmoth, without the complications of other mechanisms present in a number of insects (e.g., clap and fling mechanism [2] and exaggerated ventral flexion).

Both numerical and experimental methods pose challenges in studying the unsteady aerodynamics of bio-inspired flapping wings. As the wing size becomes smaller, the flapping frequency increases and the Reynolds number decreases. Consequently, the flowfields around the flapping wings feature formation of large-scale vortex structures closely related to the onset of separation and reattachment, lag between the instantaneous wing motion and the response of the flowfield and the aerodynamics forces, and three-dimensional effects [3]. Moreover, the fluid physics associated with the flapping wing is qualitatively and quantitatively influenced by the kinematics as well as the Reynolds number [4]. For fruit flies and honey bees with Reynolds number around $1 \times 10^{2}$ and $1 \times 10^{3}$, issues such as turbulence are less dominant. On the other side of the low-Reynoldsnumber regime between $1 \times 10^{4}$ and $6 \times 10^{4}$, several studies have assessed the fluid physics in a collaborative experimental and numerical framework $[\underline{5}, \underline{6}]$.

Multiple tools are available for the design and development of aerodynamic systems, which vary from sophisticated high-fidelity computational models solving the Navier-Stokes equations [6] to lower-fidelity reduced-order vortex models [7], to theoretical potential-flow models. In general, high-fidelity models require more computational resources and computer time compared with lowfidelity models. The performance improvement of low-fidelity models is achieved through simplifying assumptions of the flow physics that reduce the dimensionality of the computational problem and, at the same time, limit the accuracy of the results. It follows that validation of computational tools, typically by comparison with suitable experimental results, is important. Experiments used for validation also have intrinsic limitations, primarily due to facility size, sensor noise, and spatial resolution of flowfield measurements. Over the past few years, the authors have developed high- and lowfidelity computational models and advanced experimental techniques for the study of flapping-wing systems. The present research is an attempt to cross-validate these computational tools and experimental techniques by applying them to the same problem. Specifically, the goal of the present research is to provide a detailed comparison between experimental and numerical analysis of the complex flow about flapping wings in hover. A bio-inspired wing motion derived from the hawkmoth wing kinematics is used, which captures essential features of the complex flow about flapping wings [8].

The primary objective of this paper is to compare the experimental results for three hover kinematics derived from those of a hawkmoth, as presented by Vandenheede et al. [8] with the results obtained with an unsteady vortex model [7] and a high-fidelity computational fluid dynamics (CFD) code [6] that solves the Navier-Stokes equations. Thus, the accuracy and computational efficiency, in terms of computer time required, can be assessed. The Reynolds number and reduced frequency for the results presented are representative of a hawkmoth in hover (i.e., $R e=4.8 \times 10^{3}$ and $k=0.38$, respectively). A pitch-plunge apparatus is used to produce the hover kinematics. Although this is a significant change compared to actual flapping-wing kinematics, this simplification enabled the high-resolution force and velocity measurements necessary to validate the results. We primarily focus on both the time-averaged values and the time histories of the thrust generation and the corresponding vorticity field. The timeaveraged values are relevant for micro aerial vehicles, when the flapping timescale is orders of magnitude smaller than the vehicle time scale. Moreover, the instantaneous force histories are essential for performance evaluation, flight dynamics modeling, and control.

In this paper, we use a rigid wing to simplify experimental measurements and focus on the differences and similarities in the results obtained using experimental and numerical methods. The wing planform is a Zimmerman wing, which can be viewed as a rough approximation of a hawkmoth wing. It is well established that structural flexibility can significantly affect the outcome of the aerodynamics due to the coupled fluid-structural interactions. Recent studies in this area can be found in [9-11].

The outline is as follows. We first describe the case setup, followed by a description of the experimental and numerical methods in Sec. II. The comparisons of the force coefficients and the flow structures are shown and discussed in Sec. III. Finally, the conclusions are presented in Sec. IV.

\section{Methodologies}

\section{A. Scaling and Nondimensional Parameters}

Several hover kinematics, inspired by the kinematics of a hawkmoth (Agrius convolvuli), are used in this study. The process used to derive the pitch-plunge kinematics from the hawkmoth hover kinematics is described next. As a starting point for determining the relevant scaling and nondimensional parameters, the values for the hawkmoth wing motion are presented first, followed by the corresponding values for the pitch-plunge kinematics.

\section{Hawkmoth (Agrius Convolvuli)}

The Agrius convolvuli is a subspecies of the hawkmoth Sphingidae. It is a large species with a wingspan of about $80-105 \mathrm{~mm}$. Similar to hummingbirds, they have evolved to feed on nectar while hovering in front of a flower. This capability makes them an excellent study object for flapping hovering flight. The kinematics of this moth in hovering flight have been measured by Liu et al. [12], covered in Sec. II.B. The motion is relatively simple and does not include a clap and fling mechanism. The Agrius convolvuli is four-winged, but the motion of the wings on both sides in hover is nearly the same and can be assumed to act as one wing. The wing combined planform of both wings is similar to the Zimmerman planform and will be modeled as such.

The parameters determining the dynamics are presented in Table 1 . The flapping frequency and amplitude are those of the moth in hover. To calculate the relevant dimensionless parameters for hover, a reference point on the wing needs to be chosen. In general, the wing tip is used for this purpose [9]; however, the objective is to capture the most relevant phenomena and try to represent these in a pitch-plunge motion. Because the wing translates, the local wing velocity is the same for any point on a line in spanwise direction. For a flapping motion, this local velocity will scale linearly with the spanwise distance from the root. Hence, using the tip velocity will make the scaling parameters too large; a reasonable approximation is to use three quarters of the span $\left(L_{\text {ref }}=0.75 R_{m}\right)$ :

$$
U_{\text {ref }}=2 \Phi f L_{\text {ref }}
$$

The Reynolds number can then be rewritten as follows:

$$
R e=\frac{U_{\mathrm{ref}} c_{m}}{\nu_{a}}=\frac{2 \Phi f_{m} L_{\mathrm{ref}} c_{m}}{\nu_{a}}
$$

In turn the reduced frequency [Eq. (3)] is based on the same reference velocity:

$$
k=\frac{\pi f_{m} c_{m}}{U_{\text {ref }}}
$$

With the reference velocity based on three quarters of the span, the Reynolds number and reduced frequency are $4.8 \times 10^{3}$ and 0.38 , respectively.

Table 1 Agrius convolvuli parameters in hover and experiment parameters

\begin{tabular}{lcc}
\hline \hline Parameter & $\begin{array}{c}\text { Agrius convolvuli } \\
\text { value }\end{array}$ & $\begin{array}{c}\text { Experiment } \\
\text { value }\end{array}$ \\
\hline Mean chord length $c, \mathrm{~mm}$ & 18.3 & 62.4 \\
Semispan $R$, mm & 50.5 & 241.3 \\
Aspect ratio & 2.76 & 3.87 \\
Flapping frequency, Hz & 26.1 & 0.15 \\
Flapping/plunging amplitude $\Phi / h$ & $2 \mathrm{rad}$ & $129.1 \mathrm{~mm}$ \\
\hline \hline
\end{tabular}


2. Relevant Nondimensional Parameters

We consider a pitch-plunge motion; hence, the reference velocity is equal to the average velocity during half of the period, which is given in Eq. (4) and depends only on the amplitude and frequency of the motion:

$$
U_{\text {ref }}=4 f h_{0}
$$

Substitution of the reference velocity [Eq. (4)] leads to the following expression for the reduced frequency of the experiment. Note that, in the pitch-plunge case, for fixed-wing geometry, the reduced frequency is solely dependent on the amplitude of the motion:

$$
k=\frac{\pi c}{4} \frac{1}{h_{0}}
$$

Similarly the Reynolds number can be written as

$$
R e=\frac{4 c}{\nu_{w}} f h_{0}
$$

In the present study, the Reynolds number is kept constant at the value of the hawkmoth (i.e., $4.8 \times 10^{3}$ ), and the reduced frequency is kept at 0.38 .

\section{B. Hover Kinematics}

The kinematics used in this study are based on the kinematics of the hawkmoth. The rotation axis of the pitch motion coincides with the leading edge at the root of the airfoil. Three motions are considered in this study. The first hover motion ignores the elevation angle of the hawkmoth kinematics and simply converts the flapping and feathering angle, as shown in Fig. 1a. This motion will be referred to as hover motion 1, or HM1. The second hover motion (Fig. 1b) incorporates a correction in the flapping and feathering angle for variations in elevation angle. This motion will be referred to as hover motion 2, or HM2. The third motion is a pure sinusoidal motion with the same pitch and plunge amplitudes as the basic Agrius convolvuli kinematics. The sinusoidal kinematics are plotted in Fig. 1c.

\section{Experimental Setup}

\section{Water Channel and Wing Planform}

The experiments are performed in the University of Michigan water channel. The channel has a total volume of about $19 \mathrm{~m}^{3}$. The test section measures 610 by $610 \mathrm{~mm}$ and is $2.44 \mathrm{~m}$ long. The transparent test-section walls facilitate flow visualization and particle image velocimetry (PIV) experiments. The channel is capable of producing very low-turbulence flow; the free turbulence intensity is less than $1 \%$. Flow speeds can range from 5 to $40 \mathrm{~cm} / \mathrm{s}$. For this experiment, there is no flow in the channel because we are investigating a hover case.

A Zimmerman wing is used in all experiments; the wing has a chord of $79.4 \mathrm{~mm}$ and a span of $241.3 \mathrm{~mm}$. The planform shape consists of two ellipses, with the major axis equal to the span, and the midpoint of the ellipse is the quarter chord position, shown in Fig. 2a. The wing planform is laser-cut (PLS6.75, Universal Laser Systems) out of a transparent acrylic plate with a thickness of $2.75 \mathrm{~mm}$, and the wing edges are sharp right angles. The thickness of the wing is substantial, and the wing is henceforth assumed to be rigid; during the experiments, no deformation of the wing was observed. The wing is clamped and mounted on a rig with two motors, hanging vertically in the water channel, as shown in Fig. $2 \mathrm{~b}$. The motors are capable of performing pitch-plunge motions with a high degree of accuracy.

\section{Force Data Acquisition}

The force data are acquired using the ATI Mini 40 force/torque sensor mounted on top of the wing clamp. The raw sensor data are then calibrated and filtered using a Butterworth low-pass filter to reduce the noise.

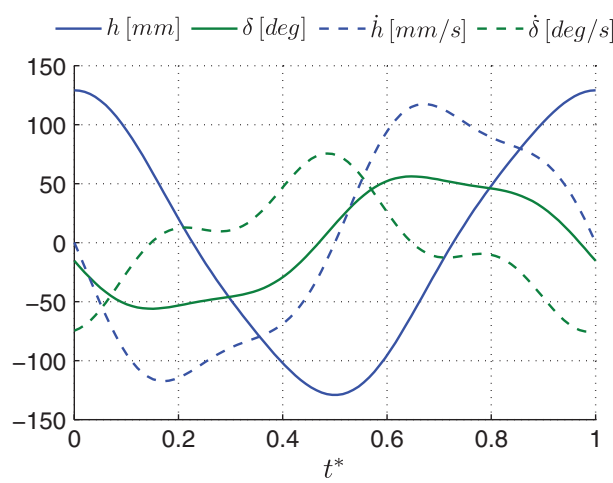

a) Pitch-plunge hover motion experiment ignoring the loss of elevation angle (hover motion 1)

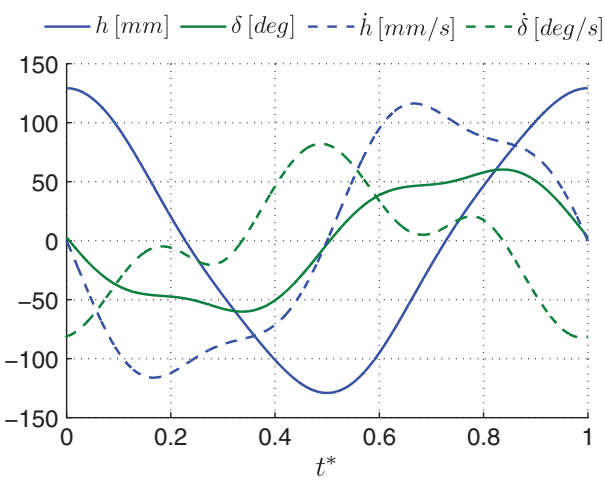

b) Pitch-plunge hover motion experiment with compensation for the loss of elevation angle (hover motion 2)

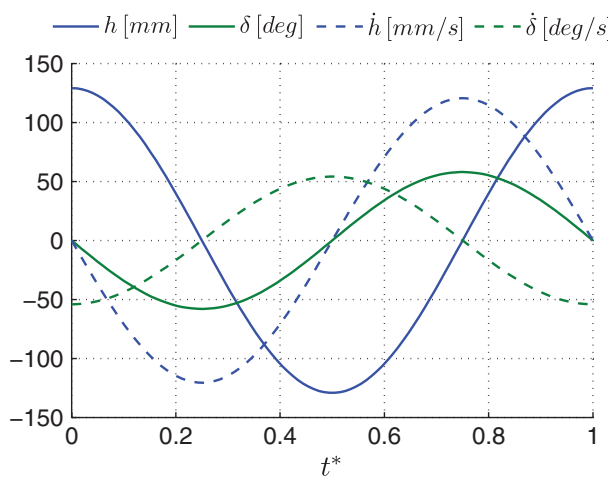

c) Pure Sinusoidal pitch-plunge motion with the same amplitude and frequency as the Agrius Convolvuli

Fig. 1 Resulting pitch-plunge kinematics of the experiments. 

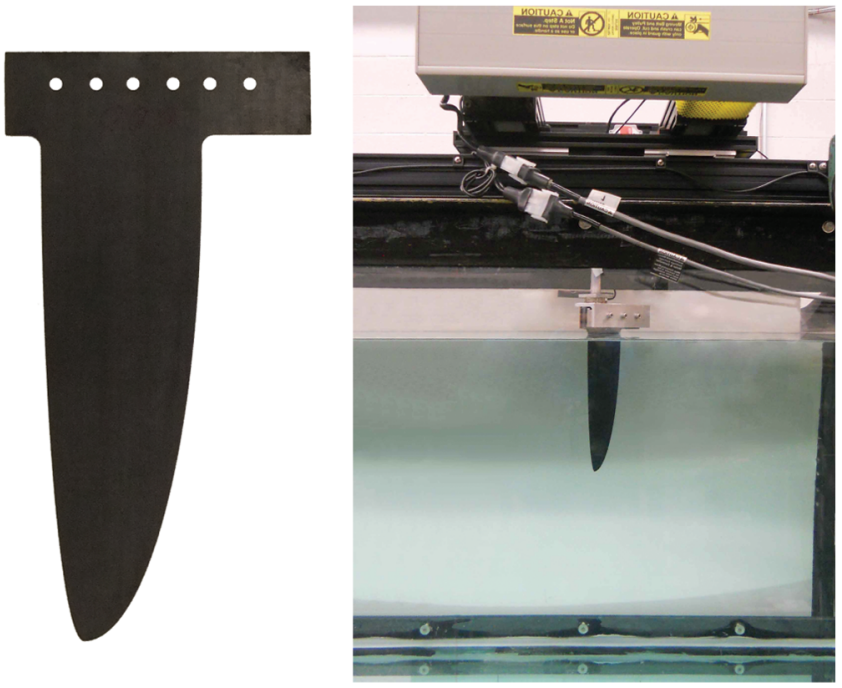

a) Wing planform

b) Wing vertically mounted in the channel

Fig. 2 Experimental setup.

Per motion, force data are acquired in six separate sets of 25 cycles. The first five cycles are cropped from each measurement, eliminating any startup phenomena that might occur. The data are then calibrated with the certified calibration matrix supplied by ATI and converted to thrust and lateral force components. The thrust is defined as the force perpendicular to the plunge motion; similarly, the lateral force is the force lateral to the plunge motion.

The thrust and lateral force components are filtered with a low-pass Butterworth filter with a cut off frequency of $3 \mathrm{~Hz}$. To eliminate the phase shift introduced by the filter, it is run over the data in the forward and reverse time direction using MATLAB's filtfilt function. This process ensures zero phase distortion and squares the magnitude of the original filter transfer function.

Next, the remaining 120 cycles are phase averaged, and the sample mean and the corresponding standard deviation are calculated. The standard deviation is good indication of the accuracy of the measurement and is plotted as an error bar in the force plots.

\section{Particle Image Velocimetry}

The PIV setup consists of a pulsed Nd:YAG laser (Spectra-Physics PIV-300), an optical setup to form the laser sheet, an external timing system (a BNC 555 pulse delay generator and the Stanford Research Systems's DG535), a high-resolution 14 bit cooled charge-coupleddevice camera system (Cooke, PCO4000) with a Nikon MicroNIKKOR $105 \mathrm{~mm}$ lens. The camera has a sensor of 4008 by 2672 pixels. The flow is seeded with Titanium(IV) oxide, rutile powder with a diameter of about $5 \mu \mathrm{m}$.

In the setup, the laser sheet is horizontal, parallel to the water surface, and the camera is underneath looking up, as illustrated in Fig. 3. The plunge amplitude is about $130 \mathrm{~mm}$; to obtain the required spatial resolution, three camera positions are used, and the data are stitched together during postprocessing. The camera is mounted on a slide and shifted to three positions that have an overlap region. The stitching procedure is completely discrete, i.e., no (weighted) averaging procedure was used in the overlap region. The discrete stitching ensures that discrepancies between images would be spotted visually. The camera lens is focused to produce a magnification of approximately 14 pixels per millimeter. Using this technique, a field of view of 6225 by 3525 pixels (or 437 by $247 \mathrm{~mm}$ ) is obtained, capturing the entire plunge motion. The time between image pairs is $2 \mathrm{~ms}$.

Similar to the force data acquisition, the PIV data are acquired over 120 cycles and processed. The postprocessing of the images was performed using in-house developed software written in MATLAB. A high-resolution interrogation window of 32 by 32 pixels is used, with a search window of 8 by 8 pixels. The calculated velocities are subsequently phase-averaged.

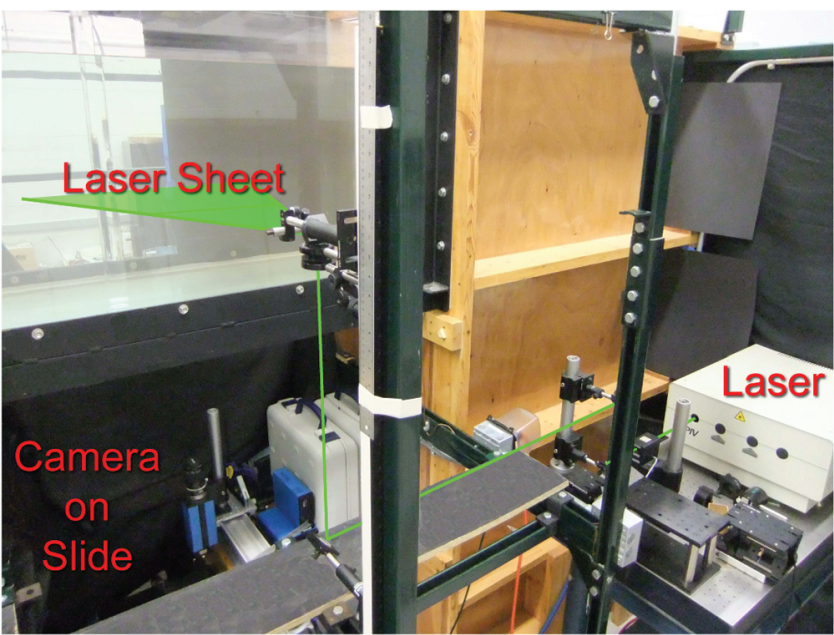

Fig. 3 Representation of the PIV setup parallel to the water surface.

\section{Unsteady Aerodynamic Vortex Model}

The unsteady aerodynamic model is based on two-dimensional potential flow and uses a vorticity/circulation approach to compute the aerodynamic loads. The formulation was originally derived for rigid wings in hover $[13,14]$. The model has been subsequently modified to account for spanwise and chordwise wing flexibility [15] and freestream due to forward flight speed [7]. The effect of the Reynolds number is also incorporated into the calculation of shed vorticity [7].

Transient and time-averaged forces, computed using the approximate model, were compared with those obtained from CFD-based computations for rigid airfoils as well as wings in hover and forward flight $[\mathbf{7}, 15]$. For all cases considered, the approximate unsteady aerodynamic loads show reasonable agreement with CFD-based results.

The overall approach is as follows. First, the wing is divided into several spanwise stations, where each section is represented as an airfoil. For each airfoil, an airfoil-wake surface that captures the airfoil degrees of freedom and approximates the geometry of the shed wake is identified. Next, the airfoil and the airfoil-wake surface are transformed to a circle in the complex plane using a conformal mapping. Thus, the airfoil-bound and shed-wake vorticity are computed on the complex plane. The quasi-steady component of vorticity is obtained by neglecting the effect of the shed wake. The strength of shed vorticity is computed by enforcing a stagnation condition at the leading edge (LE) and a Kutta condition at the trailing edge (TE). The airfoil bound vorticity is obtained as a sum of the quasi-steady and wake-induced vorticity on the airfoil. Next, the vorticity in the complex plane is transformed back to the airfoil-wake surface (physical plane) using an inverse transform. The unsteady loads acting on the airfoil are obtained from the total vorticity using the unsteady Bernoulli equation. Finally, the shed vorticity is convected using the Rott-Birkhoff equation, which is derived from the Biot-Savart law for two-dimensional flow.

For the case of hover, the vortices that are shed during previous cycles remain in the vicinity of the wing and therefore influence the forces generated by the wing even after several subsequent cycles. Consequently, the mean forces were computed once the forces reached an approximate steady state. For all of the cases considered, an approximate steady state was reached after the second cycle.

Simulations also showed that decreasing the vortex core size improved correlation with experimental results. Decreasing the vortex core size promotes vortex roll up. Thus, it appears that a core size of $0.00625 c$ captures the vortex dynamics most accurately. Furthermore, decreasing the size of the vortex core also decreased the noise generated due to interaction of the vortices with the airfoil, thereby improving the quality of the solution. It appears that decreasing the core size further had a comparatively minor impact on the forces (illustrated in Fig. 4). 


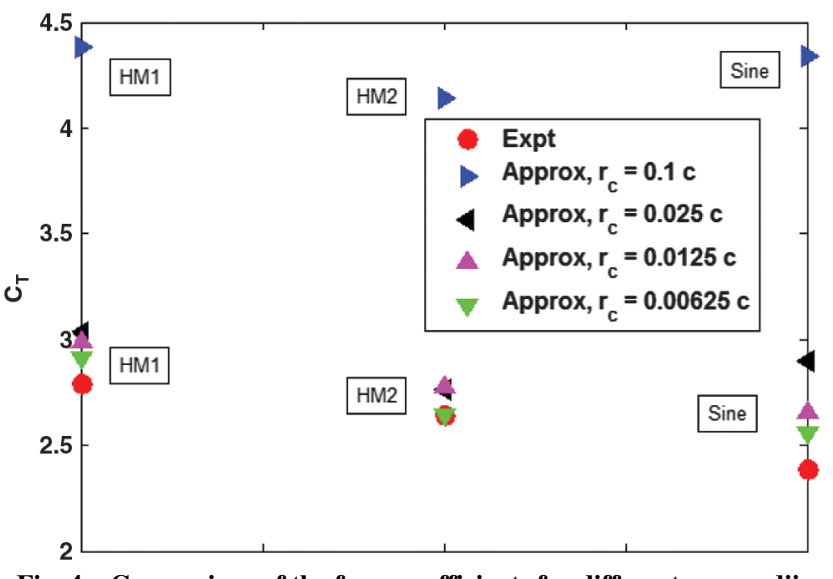

Fig. 4 Comparison of the force coefficients for different core radii.

\section{E. Computational Fluid Dynamics: Navier-Stokes Equation Solver}

The governing equations for fluid motion given by the NavierStokes equations are solved with Loci-STREAM [4,6], which is a three-dimensional, unstructured, pressure-based finite-volume solver written in the Loci framework. It employs implicit first- or second-order time stepping and treats the convection terms using the second-order upwind-type scheme and the pressure and viscous terms using second-order schemes. The system of equations resulting from the linearized momentum equations are handled with the symmetric Gauss-Seidel solver. The pressure correction equation is solved with either the generalized minimal residual linear solver with Jacobi preconditioner provided by PETSc [16-18] or the BoomerAMG [19] linear solver provided by Hypre. The Loci framework is, by design, a rule-based, highly parallelizable framework for finite-volume methods [20]. The geometric conservation law [21], a necessary consideration in domains with moving boundaries, is satisfied [22]. The motion of the wing is realized by rigidly moving the computational mesh $[\underline{4}, \underline{6}]$.

The computational grid to solve the Navier-Stokes equations consists of mixed brick and tetrahedral cells around the Zimmerman wing; see Fig. 5. To assess the grid size sensitivity, four grids with different spatial resolutions are used with $0.34 \times 10^{6}$ (blue solid), $0.51 \times 10^{6}$ (black dashed-dotted), $0.73 \times 10^{6}$ (red dashed), and $5.7 \times$ $10^{6}$ (magenta dotted) cells for each mesh. For the time-step sensitivity analysis, 250 (blue solid), 500 (red dashed), and 1000 (black dotted) time steps per motion cycle were chosen on the grid with $0.51 \times 10^{6}$ cells. From the results shown in Fig. 6 , the intermediate grid with $0.51 \times 10^{6}$ cells and 500 time steps per motion cycle show solutions with sufficient spatial and temporal resolution. The thrust on the finest mesh $\left(5.7 \times 10^{6}\right)$ has the highest peak by around $7 \%$, but the chosen mesh is able to capture the physics closely. The fluid flow is assumed to be laminar. The first grid spacing from the wing surface is set to $2.5 \times 10^{-3} c$, and the outer boundary of the computational grid is located at 30 chords away from the wing. At the outer computational boundary, zero velocity and reference density are assigned. On the wing surface, the no-slip condition is applied.

\section{Results and Discussion}

\section{A. Nondimensionalization of the Data}

The force data are nondimensionalized by the dynamic pressure (based on the reference velocity $U_{\text {ref }}$ equal to $4 f h_{0}$; see Sec. II.A.2) and the wing planform area. The thrust coefficient is then defined as shown in Eq. (7):

$$
C_{T}=\frac{T}{q S}
$$

Similarly, the lateral force coefficient is normalized by the same dynamic pressure and the projected wing surface wing planform area.

\section{B. Comparison of Force Generation}

The force coefficients, as described in Sec. III.A, for the three motions are presented in this section. The data-acquisition method for the experiment is described in Sec. II.C.2. In the experiment, the first five cycles were cropped from the data set to omit transient effects that occur. It was found that, in these few cycles, the measurement data contained more noise, attributed to the fact that vortices are not shed instantly due to the lack of a freestream flow.

For forces obtained from the Navier-Stokes (NS) computations and the unsteady aerodynamic vortex model, the first two cycles were cropped from the data set to remove the initial transient effects, and the remaining three cycles were phase averaged. The comparison of the time history of the forces is shown in Fig. 7. The unsteady aerodynamic vortex model (UAVM) takes approximately $6 \mathrm{~h}$ on 12 CPUs to compute for five motion cycles, while the Navier-Stokes computations take approximately $16 \mathrm{~h}$ on 24 Intel Xeon CPUs.

The shape of the time history of the force coefficients for the NS calculations is similar to the measurements for all motions with slight underprediction. As for the experimental results, there is a slight asymmetry in the force coefficients during the up- and downstroke. For HM1, the NS results can almost be linearly scaled to fit the experimental results. For the HM2 kinematics, the difference between the first and second maxima in the thrust coefficient is slightly larger in the NS calculations. And the differences between the up- and downstroke are less pronounced for the sinusoidal motion. For all motions, however, the shape is very similar compared to experimental results. The time of the peaks and valleys in the force coefficients are nearly the same; the maximum lag between two

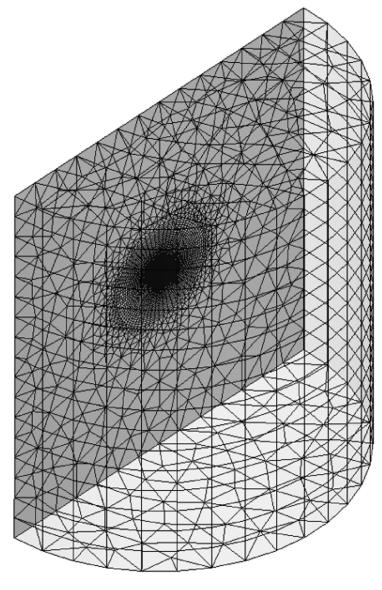

a) Computational domain

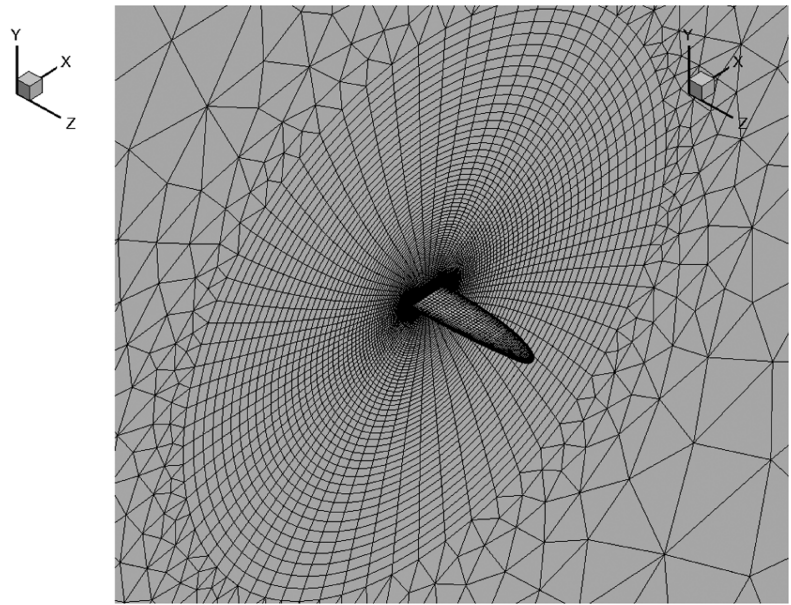

b) Mesh distribution around the Zimmerman wing

Fig. 5 Computational grids for the Zimmerman wing. 


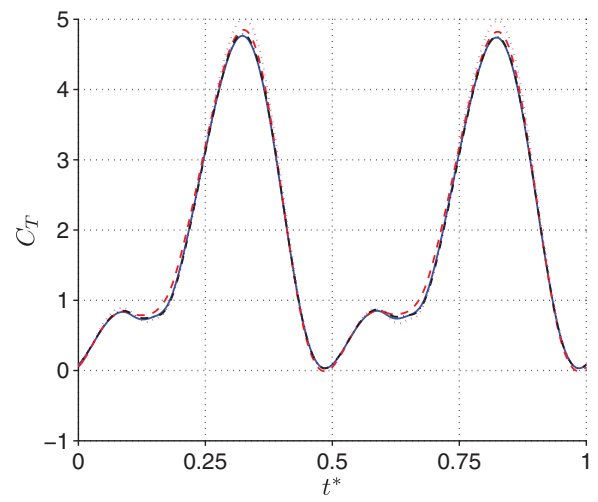

a) Spatial

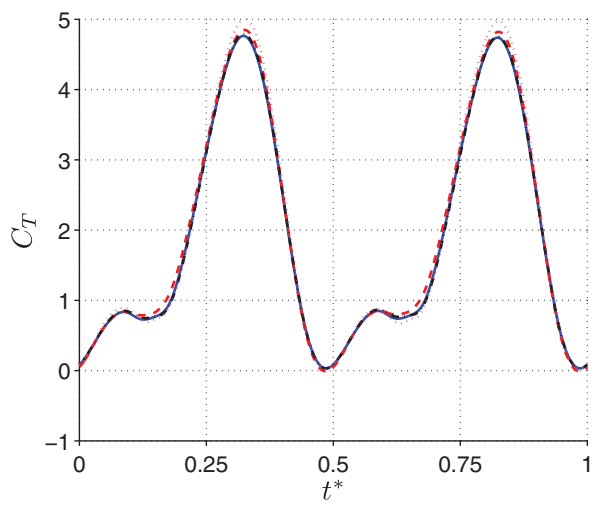

b) Temporal
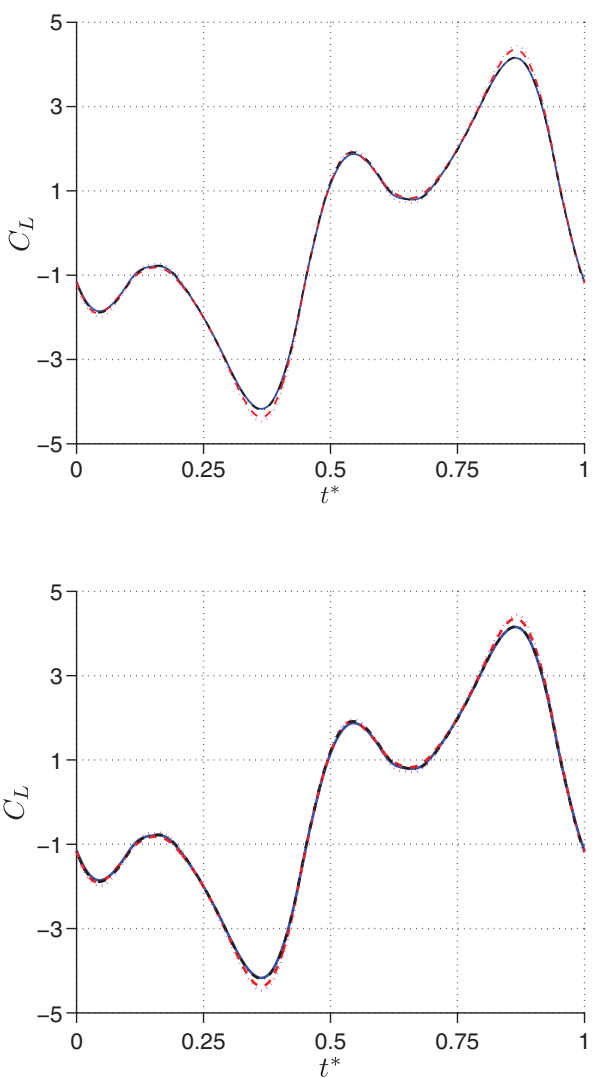

Fig. 6 Spatial and temporal sensitivity tests for the pure sinusoidal motion.

maxima is $1.8 \%$ percent of the period, which is found for the HM1 motion.

The unsteady aerodynamic model predicts a shape of the time history of the force coefficients that is less similar to the experiments. The unsteady aerodynamic vortex model underpredicts the force coefficients of the experiment at the beginning of the stroke, $t^{*}=0-0.2$, and overpredicts the force coefficients toward the end of the stroke, generally for $t^{*}=0.3-0.5$, and similarly for the reverse stroke. This trend is observed for all three motions. The locations of the peaks are accurately predicted by the model; the maximum lag between two maxima is $5.1 \%$ of the period and occurs for HM2 kinematics.

The average force coefficients for the experiment, the NS calculations (averaged over the last four cycles), and unsteady aerodynamic vortex model (averaged over the last four cycles) are summarized in Table 2 . The average force coefficients, computed and measured, are of the same order of magnitude and the differences are always smaller than $31 \%$ with respect to the experiment.

On average, the force coefficients computed with NS are between 82 and $87 \%$ of the values measured in the experiment; the NS computations underpredict the force coefficients consistently, as shown in the time histories in Fig. 7. The underprediction can also be seen as the difference in slope between the NS computations and the experimental results shown in Fig. 8. Possible reasons for these differences may be found in the boundary conditions of each setup. First, in the experimental setup, the water channel walls are relatively close to the wing at some phases of the motion, which may result in blockage effects. In the NS computations, the outer boundary of the computations domain is located orders of magnitude away from the motion. Blockage effects would increase the forces measured. Second, in the experiments, an offset between the center of the motions and the center of the water channel could cause an asymmetry in the measured forces during the up- and downstroke. The minimum distance to the wall was 2.5 chords. The flow in the NS computation is assumed to be fully laminar without an attempt to resolve small turbulence scales to capture turbulence or laminar-toturbulence transition. Another possible reason for the discrepancy is difference in the flow induced by the thrust-producing wing. The experimental results are averages over 120 cycles to produce a well-established induced flow in the facility. In contrast, the computational results converge to a stable solution in only a few cycles are only over a few cycles. These potential sources of disagreement between the experiment and CFD have not been quantified and are being investigated.

Similar to predicting the shape of the time history of the forces, the prediction of the average force coefficients by the unsteady aerodynamic model is also less accurate. The difference in averaged force coefficients for HM1 and HM2 is about $20 \%$, about $7.5 \%$ for the thrust coefficient of the pure sinusoidal motion, and $31 \%$ for the lateral force coefficient. These time-averaged thrust coefficients are closer to the experimental values compared to the NS computations, due to cancellation effects with an underprediction in the first part of the stroke, $t^{*}=0.05-0.25$, and overprediction of the forces in the second part of the stroke, $t^{*}=0.3-0.5$. Potential sources of these discrepancies are discussed in Sec. III.C. The standard deviation, Std $C_{T}$ (Table 2), for the unsteady aerodynamic vortex model is indeed much greater than for the NS computations. Note that $\operatorname{Std} C_{T}$ reduces when the time history of thrust coefficient is corrected for the phase difference. A more detailed comparison between experiments and computations is obtained by plotting the experimental results against the computational results, as shown in Fig. 8 for the thrust coefficient and the three kinematics. The solid straight lines at $45 \mathrm{deg}$ represent perfect agreement. The NS results align along a narrow region oriented at an angle smaller than $45 \mathrm{deg}$. This supports the fact that both the NS computations and experiments capture the same flow physics and that the small differences can be attributed to changes between the setups, as noted earlier. In contrast, comparison between the vortex method and experiments show more fundamental differences. The peak values of the thrust coefficients occur at a different phase in the computations and experiments, resulting in large departures from the $45 \mathrm{deg}$ straight line. There are also differences in the evolution between maxima. For HM1 kinematics, the double oval shape is due to the three maxima in the computational results 


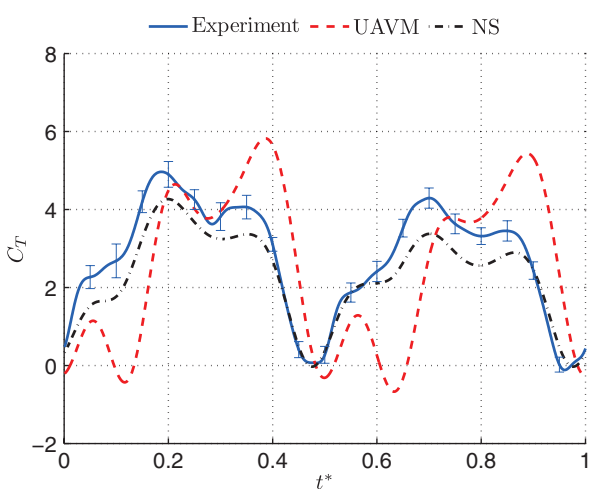

a) Thrust coefficient hover motion 1

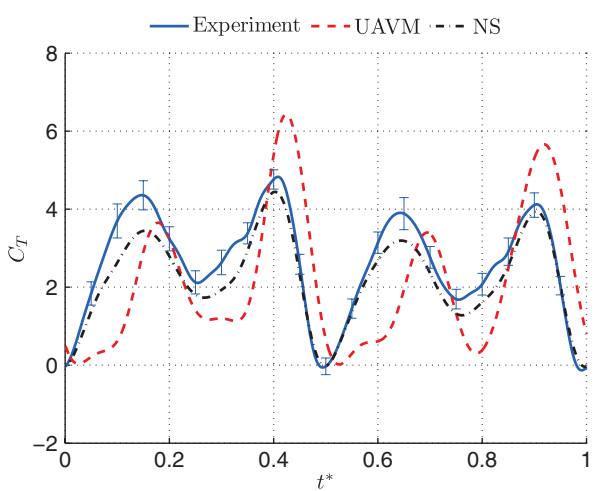

c) Thrust coefficient hover motion 2

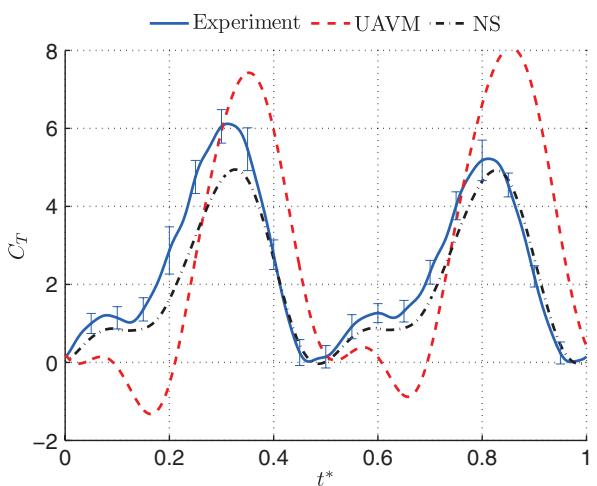

e) Thrust coefficient pure sinusoidal motion

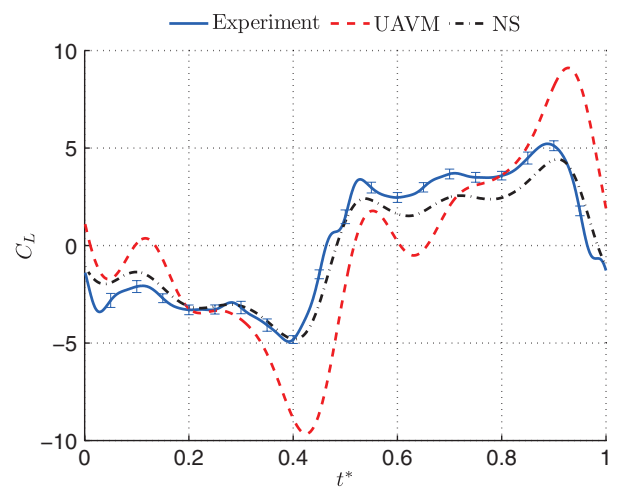

b) Lateral force coefficient hover motion 1

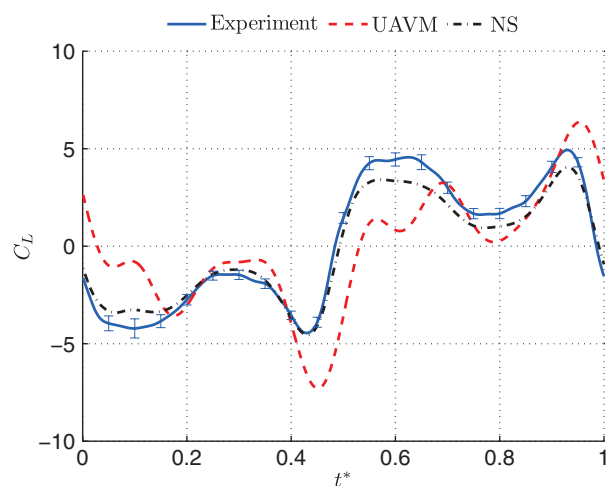

d) Lateral force coefficient hover motion 2

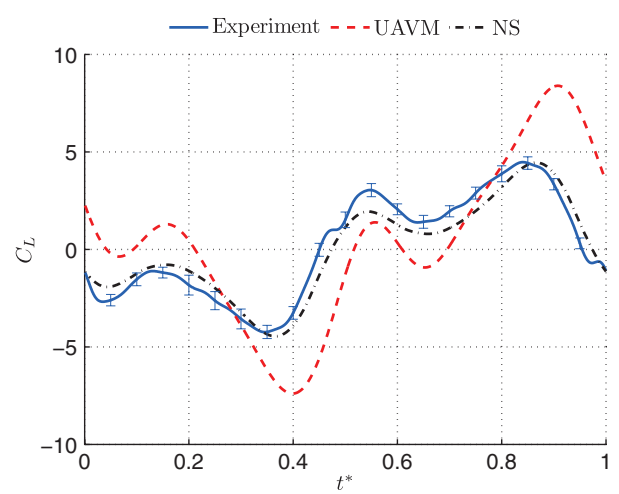

f) Lateral force coefficient pure sinusoidal motion

Fig. 7 Comparison of the force coefficients of all three motions for the experiment (the error bar is one standard deviation), unsteady aerodynamic vortex model, and the NS computations.

compared to two maxima in the experimental measurements in each stroke. For HM2 kinematics, a loop is found between the maxima. In this case, there are two maxima in both computations and experiments. In contrast, for the sinusoidal kinematics, the large second maxima results in a single oval shape. Differences between the upand downstroke are also observed, although the overall shape is the same for each kinematic. Clearly, the UAVM overpredicts the amplitude and phase of force maxima. To gain a better understanding of these differences, we examine the vorticity field for each of the kinematics in the next section.

Three-dimensional spanwise flow should contribute to the differences in force history between the unsteady vortex model and the CFD and experimental measurements. Flow visualization data [23] and the flowfield obtained from the NS computations reveal the presence of tip vortices and a spanwise component of the flow from the tip toward the root of the wing. Compared to the tip vortices on a

Table 2 Comparison of the averaged force coefficients and standard deviation

\begin{tabular}{lccccccccc}
\hline \hline & \multicolumn{3}{c}{ HM1 } & \multicolumn{3}{c}{ HM2 } & \multicolumn{3}{c}{ Sine } \\
\cline { 2 - 10 } & Exp & NS & UAVM & Exp & NS & UAVM & Exp & NS & UAVM \\
\hline$C_{T}$ & 2.79 & 2.31 & 2.54 & 2.64 & 2.23 & 2.17 & 2.39 & 1.97 & 2.57 \\
Mean $\Delta C_{T}$ & -- & -0.475 & -0.243 & -- & -0.41 & -0.479 & -- & -0.421 & 0.184 \\
Std $C_{T}$ & -- & 0.379 & $1.84\left(1.25^{\mathrm{a}}\right)$ & -- & 0.0295 & $1.56\left(1.01^{\mathrm{a}}\right)$ & -- & 0.499 & $2.21\left(1.55^{\mathrm{a}}\right)$ \\
\hline \hline
\end{tabular}

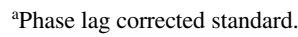




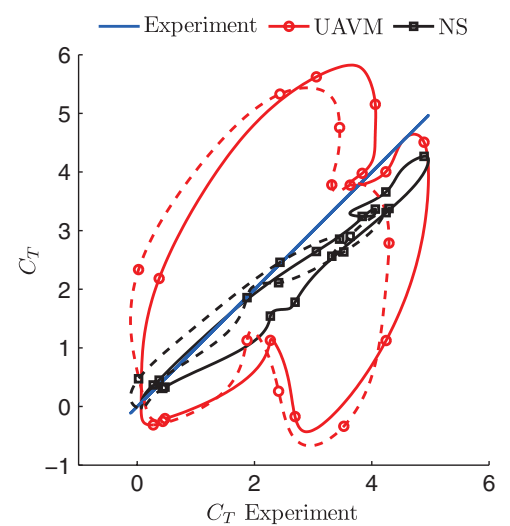

a) Hover motion 1

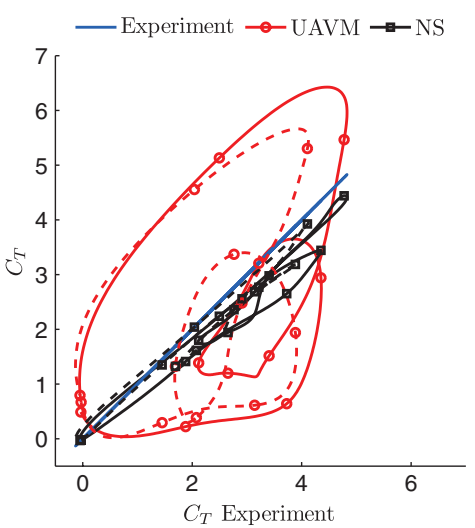

b) Hover motion 2

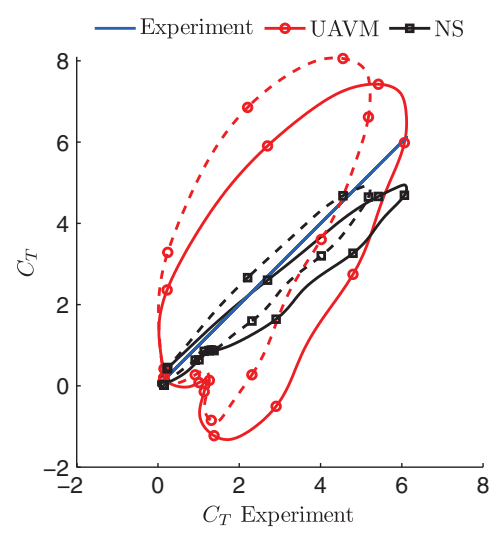

c) Sinusoidal motion

Fig. 8 Correlation plot of the force coefficients of all three motions. The solid lines and dashed lines represent the up and down-stroke respectively.

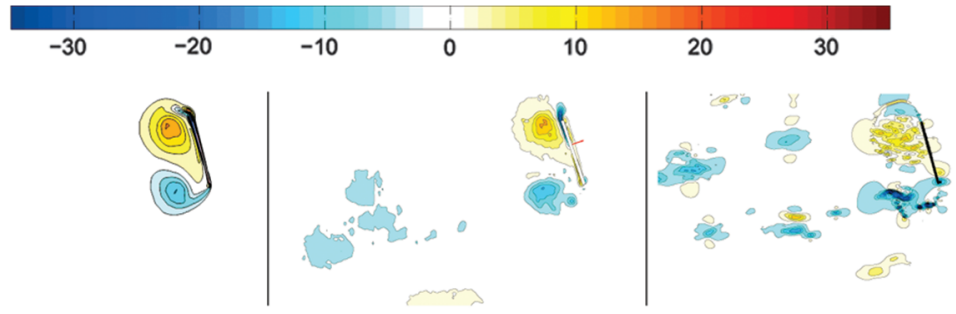

a) $t^{*}=0.0$

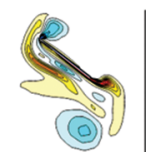

b) $t^{*}=0.1$

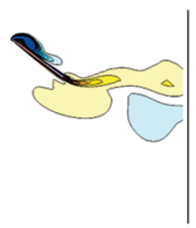

c) $t^{*}=0.2$

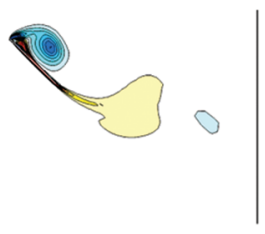

d) $t^{*}=0.3$

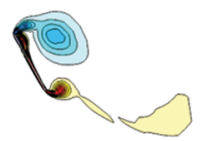

e) $t^{*}=0.4$

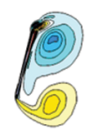

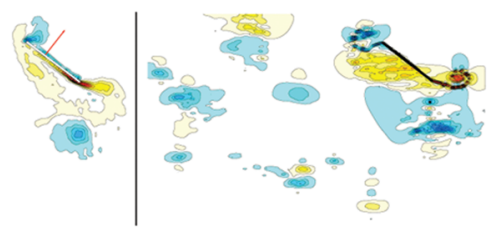
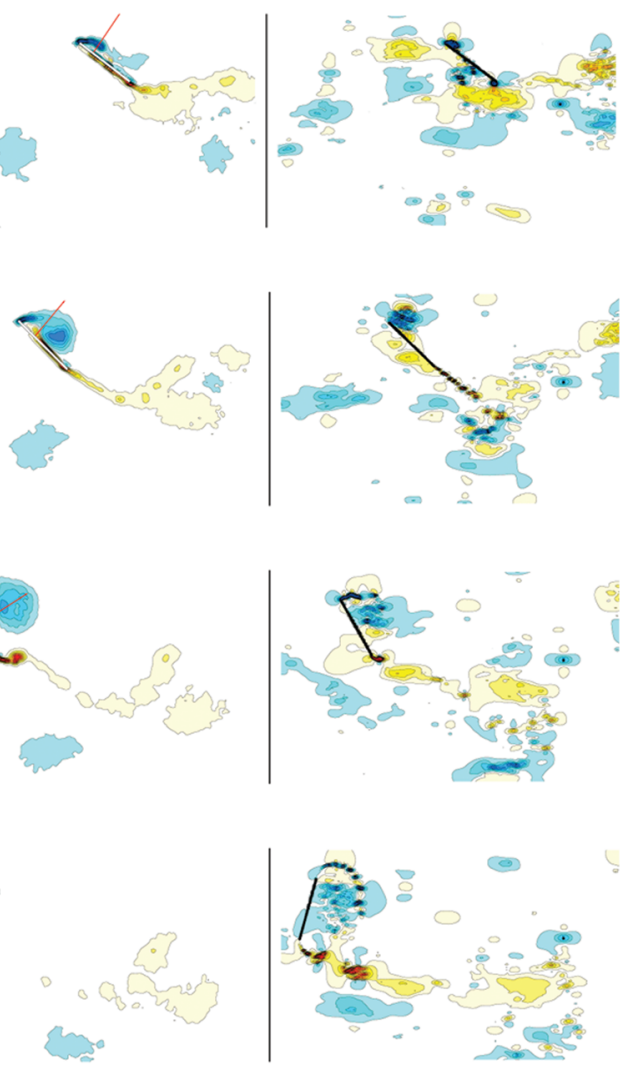

f) $t^{*}=0.5$

Fig. 9 Comparison of vorticity plots for HM1 base at half-span. From left to right: the NS computation, the PIV experiment, and the unsteady aerodynamic vortex model. 
hovering flat plate at lower Reynolds numbers (i.e., $R e=100$ ), the sizes of the tip vortices observed in this study were smaller, also due to the narrower wing shape near the tip. On the other hand, Shyy and Liu [24] investigated the role of leading-edge vortices (LEVs) and spanwise effects at $R e$ ranging from 10 to 6000 for insectlike wings and kinematics. They observed that the spanwise effects were the greatest at $R e=6000$ for hawkmoth kinematics, and the spanwise flow became weaker as the Reynolds number is lowered. More detailed and concrete study of the three-dimensional spanwise effects as a function of $R e, k$, and kinematics is left as future study.

\section{Comparison of Vorticity Field}

Complementary to the force data, the vorticity fields were measured and computed. To attain the vorticity field in the experiment, two-dimensional particle image velocimetry is performed. From the experiments, it was found that the flow topology at half-span is representative for the flow topology on the majority of the airfoil [23].

The vorticity field reveals a number of large-scale vortical features (e.g., formation of a leading-edge vortex). These large-scale features are observed by all three methods and differ distinctly for all three motions.

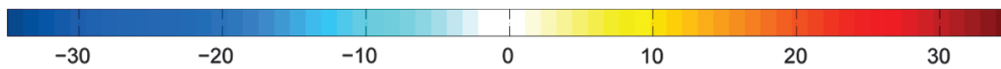

a) $t^{*}=0.0$
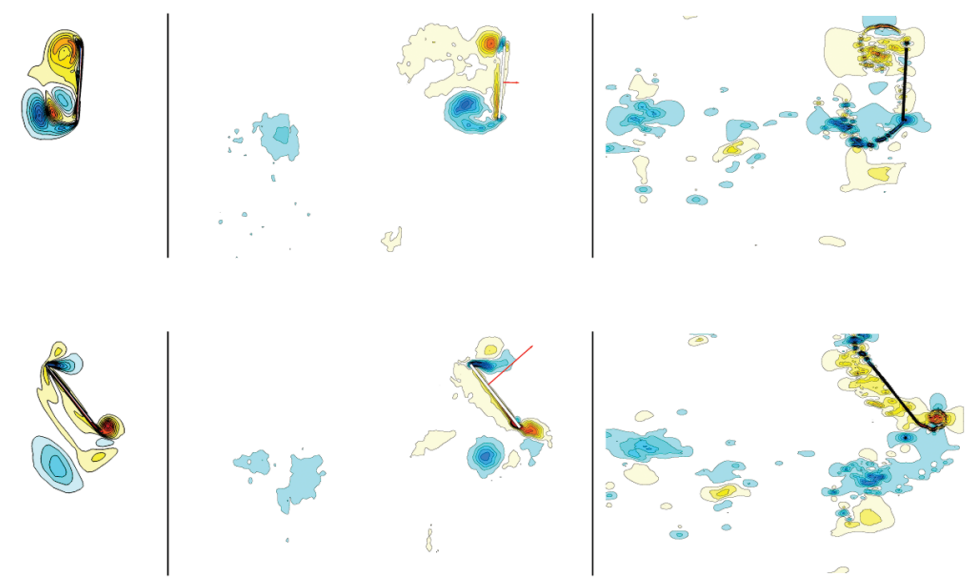

b) $t^{*}=\mathbf{0 . 1}$
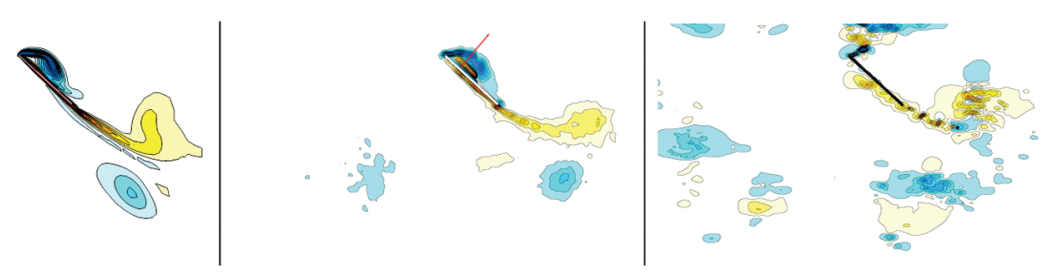

c) $t^{*}=0.2$
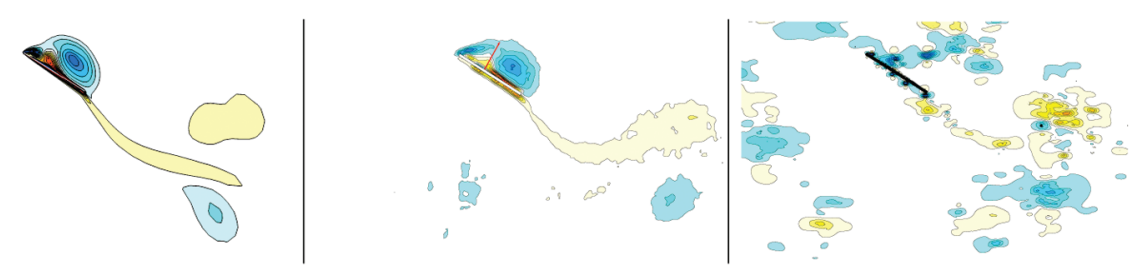

d) $t^{*}=0.3$
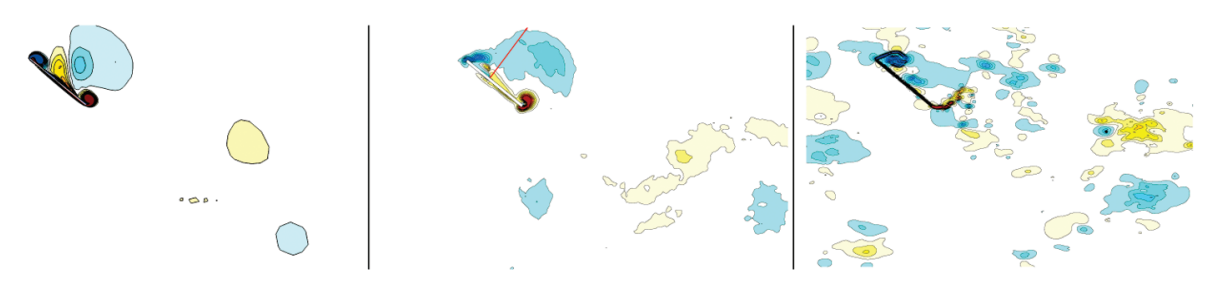

e) $t^{*}=0.4$
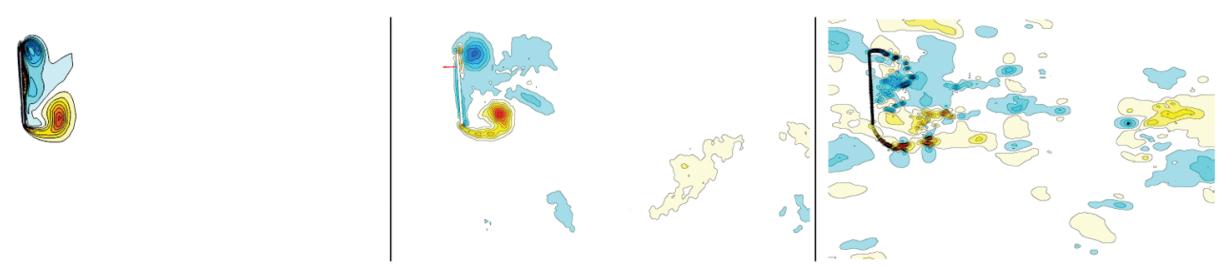

f) $t^{*}=0.5$

Fig. 10 Comparison of vorticity plots for HM2 base at half-span. From left to right: the NS computation, the PIV experiment, and the unsteady aerodynamic vortex model. 
Figure 9 shows the vorticity of the phase-averaged flowfield for HM1 at half-span and phases as indicated for the experiment, the NS computations, and the unsteady aerodynamic vortex model. At the beginning of the stroke, the LEV from the previous half-stroke interacts with the wing and breaks into two: a small part flowing over the leading edge and dissipating almost immediately, and a larger part that moves along the airfoil surface to the trailing edge, where it interacts with the trailing-edge vortices (TEVs). An LEV develops from approximately $t^{*}=0.2$ until the end of the stroke. The largest thrust coefficient occurs at the first peak, $t^{*}=0.2$. During wing rotation between $t^{*}=0.3$ and 0.6 , a TEV forms with counterclockwise vorticity for the wing at the left end of the stroke. During this process, the second maximum of the force coefficient occurs, at $t^{*}=0.4$. This vortex and a second TEV, a starting vortex having opposite circulation that forms at stroke reversal, combine to form a persistent vortex structure.
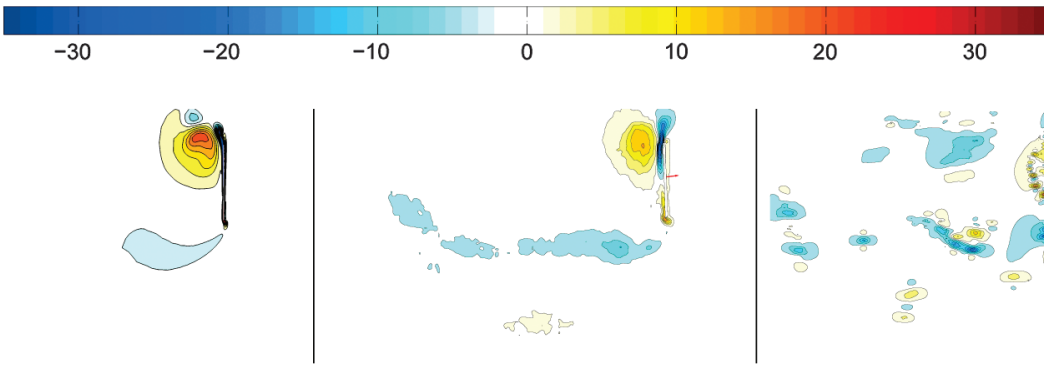

a) $t^{*}=\mathbf{0 . 0}$
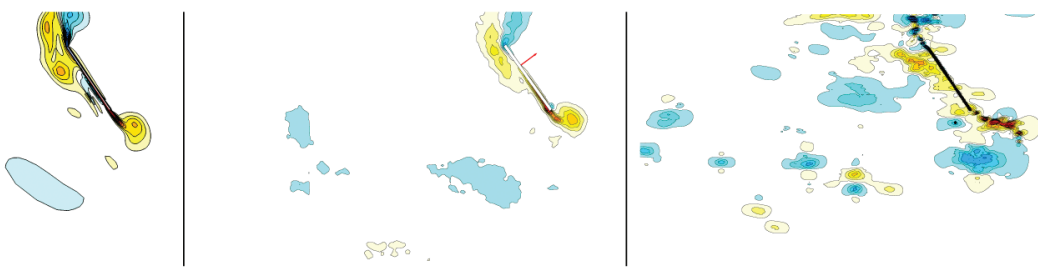

b) $t^{*}=0.1$
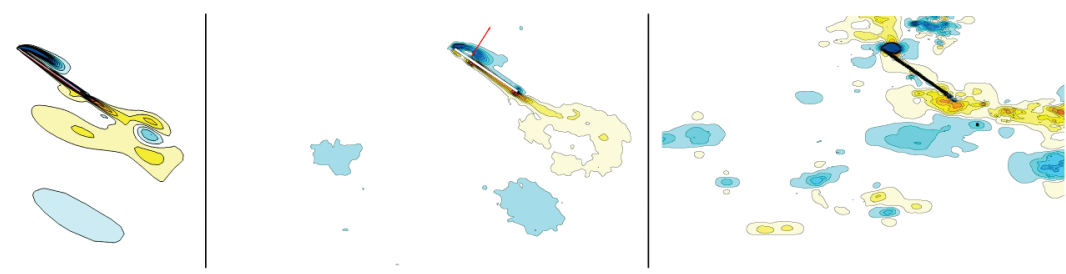

c) $t^{*}=0.2$
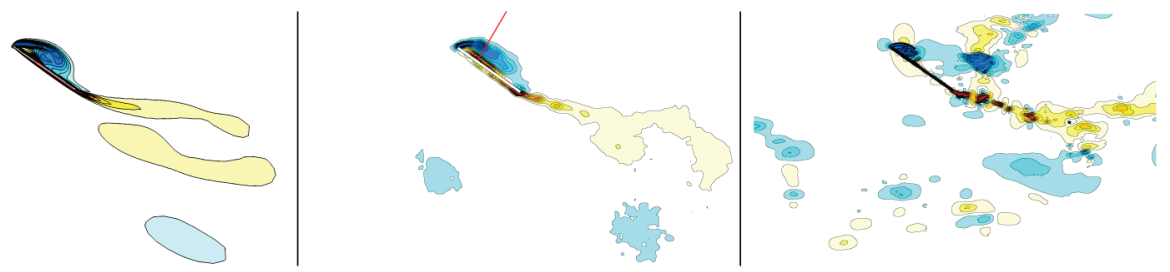

d) $t^{*}=0.3$
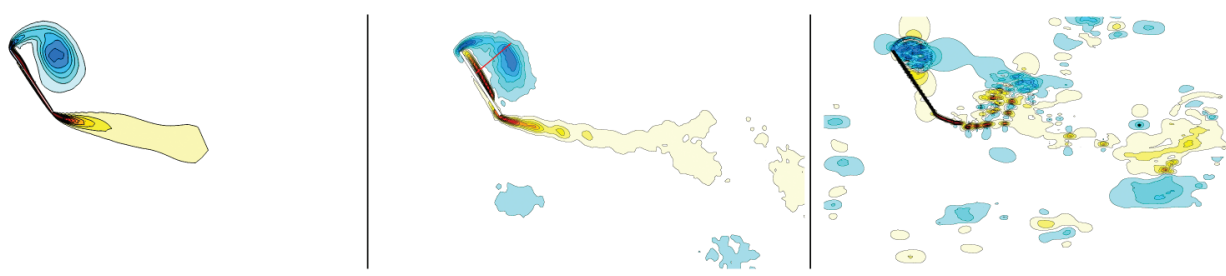

e) $t^{*}=0.4$

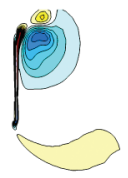

$$
\mid
$$
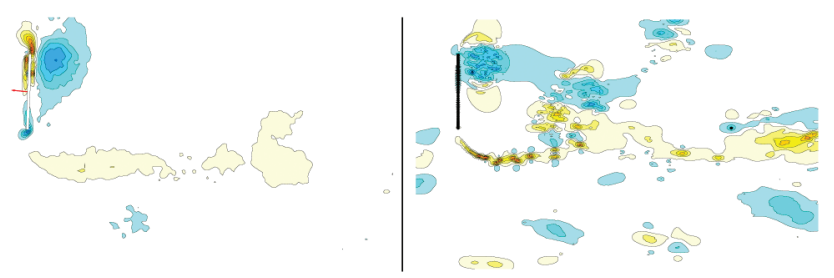

f) $t^{*}=0.5$

Fig. 11 Comparison of vorticity plots for sine base at half-span. From left to right: the NS computation, the PIV experiment, and the unsteady aerodynamic vortex model. 
The phase-averaged vorticity for HM2 is plotted in Fig. 10. Similar to HM1, the LEV interacts with the wing, right after the stroke reversal. The LEV breaks into two parts, where the larger part joins the starting vortex at the trailing edge. The other part remains around the leading edge and is entrained and annihilated by the new LEV having opposite circulation. In this case, the LEV detaches between $t^{*}=0.2$ and 0.4 , stimulated by the increase of the pitch angle at $t^{*}=0.25$. A new LEV forms around $t^{*}=0.4$. These flow features correlate closely with the two peaks in force coefficients. The first peak at $t^{*}=0.15$ in the time history of thrust in Fig. 7e is called the wake-capture peak [25], where the wing-wake interaction results in thrust enhancement. The second thrust peak $t^{*}=0.4$ is mainly due to the generation of the LEV on the suction side of the wing [25].

In Fig. 11, the phase-averaged vorticity for the sine motion are plotted. At the start of the stroke during stroke reversal, the vorticity contours show significantly weaker LE and TE vortices compared to the other two kinematics, which is consistent with the relative low force coefficient measured at these phases. For this kinematics, the LEV is observed first at $t^{*}=0.2$. The force coefficients at the beginning of the stroke are small, but as the LEV grows larger, the force coefficients surmount the maximum coefficients of both HM1 and HM2. There is no evidence of formation of TE vortices at stroke reversal, in contrast to HM1 and HM2.

The NS computations of the vorticity field compare well to the experiment. The largest difference occurs at $t^{*}=0.1$; at this phase, there is a negative vorticity region on the bottom of the airfoil that is not present in the PIV measurements. A possible reason for this difference is that the vortex, located upstream of the wing, in the computations is stronger than in the experimen; the maximum positive vorticity in that region is roughly twice as large as the experiment (Fig. 11a). As a result, when the wing interacts with this vortex at $t^{*}=0.1$, the stronger vortex in the computations leads to generation of a small secondary vortex of opposite direction, while we do not observe such a secondary vortex in the experiment. For all other phases, the vorticity field in the near field, within 1 chord length of the airfoil, is nearly identical to the measurements; further away from the airfoil, the NS computations display larger diffusion of the vorticity compared to the experiment, which may be due to numerical viscosity or blockage effects in the experiments.

The vorticity field for the UAVM is obtained first by calculating the induced velocity field from the position and strength of discrete vortices in the flowfield. The vorticity then follows by taking the curl of the induced velocity. In general, the UAVM is able to capture the major flow structures around the wing; the LEV during the midstroke as well as TEVs are well defined. However, the LEV strength is overpredicted, partly due to the assumption of constant shedding of vorticity at the leading edge. Moreover, the viscous diffusion, externally modeled into the UAVM, is less than in the NS computation or in the experiments. Finally, because of the boundary discretization effects in the UAVM, vortices in the flowfield can pass through the wing. These effects combine to produce the large difference in forces during the first part of the stroke, as depicted in Fig. 7 between $t^{*}=0.05$ and 0.15 for HM1 and the sine motion. The vorticity field at $t^{*}=0.2$ shows vortices upstream of the wing for the UAVM results that are not present in the PIV measurements and NS computations. Note also a small blob of vorticity on the suction side of the wing near the TE at $t^{*}=0.1$ in the UAVM that seems to be connected to the vorticity on the pressure side of the wing. On the other hand, for $\mathrm{HM}$ 2, the vorticity field obtained from $\operatorname{UAVM}\left(t^{*}=0.1\right)$ is closer to that of the PIV and NS computation. This is also consistent with the better correlation of the forces around this time instant for HM2. Exact quantification of these artificial effects requires future study.

\section{Conclusions}

This paper assesses the force generation and the flowfield of a hovering three-dimensional Zimmerman wing undergoing bio-inspired kinematics obtained using force and particle-image-velocimetry vorticity measurements, a Navier-Stokes equation solver, and an unsteady aerodynamic vortex model. Three pitch-plunge motions derived from measured hawkmoth kinematics are considered, namely pitch angle equal to 1) feathering angle without elevation angle (HM1), 2) feathering angle with a correction for the elevation angle (HM2), and 3) a sinusoidal fit of the feathering angle (sine).

Aerodynamic forces calculated by solving the Navier-Stokes equations are consistent with the experimental force measurements. The time-averaged computed forces are 82 to $87 \%$ of the experimentally measured forces. The small differences can be related to a number of factors. First, blockage effects in the water channel, which might increase the forces measured in the experiment. Second, the presence of a thrust-induced flow, which may not be well-developed in the simulations and may also result in higher force coefficients. Third, the Navier-Stokes equation solutions are solved assuming laminar flow; therefore, transition from laminar-to-turbulence and turbulence effects are not included but may be present in the experiments. Similar to the force results, the vorticity field computed from Navier-Stokes equation solver shows qualitatively similar flow structures. The vorticity magnitude is slightly smaller than what has been observed in the PIV measurements. The computational time for five motion cycles is about $16 \mathrm{~h}$ on 24 CPUs.

The unsteady aerodynamic vortex model captures the major trends of the force history with larger differences in the amplitude and phase than the Navier-Stokes computations. The computational time for five motion cycles is about $6 \mathrm{~h}$ on $12 \mathrm{CPUs}$. For the three cases considered, the unsteady aerodynamic vortex model underpredicts the thrust in the first half of the motion stroke, while the thrust in the second half is much greater. The resulting vorticity fields show that the strength of the leading-edge vortex is overpredicted, which in turn affects the wing-wake interaction that occurs right after the stroke reversal. Further potential sources of this discrepancy are the simplified model of vortex diffusion and the artificial wing-wake interaction. As a result, the time-averaged thrust coefficients from the unsteady aerodynamic vortex model yield closer values than the Navier-Stokes results compared to the force measurements due to cancellation effects for HM1 and sine kinematics. The difference is greater for HM2, where the vorticity field shows the least wing-wake interaction and the best agreement with the experiment and the Navier-Stokes computations. The standard deviation is consistently greater than that of the Navier-Stokes computations.

In closing, the significant differences in implementation of the same flow problem between the experiments and the high- and lowfidelity models were noted. In the experiments, reducing sensor noise dictated the use of a very large number of cycles in the phase averages. This led to relatively long duration experiments and the need to achieve stable thrust-induced flow in the facility. Also, testsection geometry is important in the resulting induced flow. These effects are generally referred to as blockage effects. The present results suggest that these effects are important, and better agreement with computational results requires advances in sensor technology or a larger facility or both. In contrast with the long-duration experiments, it is not realistic to run the numerical models for many cycles. In this case, the issue becomes how few cycles are required to achieve stable thrust-induced flow in the numerical simulations. The present results suggest that as few as three cycles are sufficient. This very small number may be due to much smaller uncertainty in the thrust-induced flow compared to experiments. In the high-fidelity simulations, better agreement with experiment may be obtained using a far-field geometry representative of the flow facility. In the low-fidelity simulations, the major source of error is implementation of the boundary conditions at the wing surface, which resulted in significant differences in the flowfield and aerodynamic force. Improvements in the low-fidelity model require a more accurate implementation of the wing boundary conditions as well as capturing three-dimensional effects. The additional computational resources and computational time required by these changes may not be justified depending on the application.

\section{Acknowledgments}

This research was sponsored in part by the U.S. Air Force Office of Scientific Research Multidisciplinary University Research Initiative contract number FA9550-07-1-0547, Doug Smith program monitor. 


\section{References}

[1] Willmott, A. P., and Ellingtion, C. P., "The Mechanics of Flight in the Hawkmoth Manduca Sexta," Journal of Experimental Biology, Vol. 200, No. 21, 1997, pp. 2705-2722.

[2] Miller, L. A., and Peskin, C. S., "A Computational Fluid Dynamics of 'Clap and Fling' in the Smallest Insects,' Journal of Experimental Biology, Vol. 208, No. 2, 2005, pp. 195-212. doi:10.1242/jeb.01376

[3] Shyy, W., Lian, Y., Tang, J., Viieru, D., and Liu, H., Aerodynamics of Low Reynolds Number Flyers, Cambridge Univ. Press, Cambridge, England, U.K., 2008, pp. 1-158.

[4] Trizila, P., Kang, C., Aono, H., Shyy, W., and Visbal, M., "LowReynolds-Number Aerodynamics of a Flapping Rigid Flat Plate," AIAA Journal, Vol. 49, No. 4, 2011, pp. 806-823. doi:10.2514/1.J050827

[5] Ol, M., Bernal, L. P., Kang, C., and Shyy, W., "Shallow and Deep Dynamic Stall for Flapping Low Reynolds Number Airfoils," Experiments in Fluids, Vol. 46, No. 5, 2009, pp. 883-901. doi:10.1007/s00348-009-0660-3

[6] Kang, C., Aono, H., Baik, Y., Bernal, L., and Shyy, W., "Fluid Dynamics of Pitching and Plunging Flat Plate at Intermediate Reynolds Numbers," AIAA Journal, Vol. 51, No. 2, 2013, pp. 315-329. doi:10.2514/1.J051593

[7] Gogulapati, A., and Friedmann, P. P., "Approximate Aerodynamic and Aeroelastic Modeling of Flapping Wings in Forward Flight," AIAA Journal, Vol. 52, No. 1, 2014, pp. 212-218. doi:10.2514/1.J052596

[8] Vandenheede, R. B. R., Bernal, L. P., Morrison, C. L., and Humbert, S., "Force Generation of Bio-inspired Hover Kinematics," 50th AIAA Aerospace Sciences Meeting, AIAA Paper 2012-0708, Jan. 2012.

[9] Shyy, W., Aono, H., Chimakurthi, S., Trizila, P., Kang, C., Cesnik, C., and Liu, H., "Recent Progress in Flapping Wing Aerodynamics and Aeroelasticity," Progress in Aerospace Sciences, Vol. 46, No. 7, 2010, pp. 284-327. doi:10.1016/j.paerosci.2010.01.001

[10] Ramananarivo, S., Godoy-Diana, R., and Thiria, B., "Rather Than Resonance, Flapping Wing Flyers may Play on Aerodynamics to Improve Performance," Proceedings of the National Academy of Sciences, Vol. 108, No. 15, 2011, pp. 5964-5969. doi:10.1073/pnas.1017910108

[11] Kang, C., Aono, H., Cesnik, C., and Shyy, W., "Effects of Flexibility on the Aerodynamic Performance of Flapping Wings," Journal of Fluid Mechanics, Vol. 689, Dec. 2011, pp. 32-74. doi: $10.1017 / \mathrm{jm} .2011 .428$

[12] Liu, H., Ellington, C. P., Kawachi, K., van den Berg, C., and Wellmott, A. P., "A Computational Fluid Dynamic Study of Hawkmoth Hovering," Journal of Experimental Biology, Vol. 201, No. 4, 1998, pp. 461477.

[13] Ansari, S. A., Zbikowski, R., and Knowles, K., "Non-Linear Unsteady Aerodynamic Model for Insect-Like Flapping Wing in Hover. Part 1: Methodology and Analysis," Proceedings of the Institution of Mechanical Engineers, Part G: Journal of Aerospace Engineering,
Vol. 220, No. 2, 2006, pp. 61-83. doi:10.1243/09544100JAERO49

[14] Ansari, S. A., Zbikowski, R., and Knowles, K., "Non-Linear Unsteady Aerodynamic Model for Insect-Like Flapping Wing in Hover. Part 2: Implementation and Validation," Proceedings of the Institution of Mechanical Engineers, Part G: Journal of Aerospace Engineering, Vol. 220, No. 2, 2006, pp. 169-186. doi:10.1243/09544100JAERO50

[15] Gogulapati, A., Friedmann, P. P., Kheng, E., and Shyy, W., "Approximate Aeroelastic Modeling of Flapping Wings in Hover," AIAA Journal, Vol. 51, No. 3, 2013, pp. 567-583. doi:10.2514/1.J051801

[16] Balay, S., Gropp, W. D., McInnes, L. C., and Smith, B. F., "Efficient Management of Parallelism in Object Oriented Numerical Software Libraries," Modern Software Tools in Scientific Computing, Birkhauser Boston, Cambridge, MA, 1997, pp. 163-202.

[17] Balay, S., PETSc Users Manual, Argonne National Lab., Lemont, IL, 2010.

[18] Balay, S., Brown, J., Buschelman, K., Gropp, W. D., Kaushik, D., Knepley, M. G., McInnes, L. C., Smith, B. F., and Zhang, H., "PETSc Web Page," 2011, http://www.mcs.anl.gov/petsc [retrieved 1 March 2011].

[19] Flagout, R., and Yang, U., "Hypre: A Library of High Performance Preconditioners," Proceedings of Computational Science - ICCS 2002, edited by Sloot, P., Hoekstra, A., Tan, C., and Dongarra, J., SpringerVerlag, Berlin, 2002, pp. 632-641.

[20] Luke, E. A., and George, T., "Loci: A Rule-based Framework for Parallel Multi-Disciplinary Simulation Synthesis," Journal of Functional Programming, Vol. 15, No. 3, 2005, pp. 477-502. doi:10.1017/S0956796805005514

[21] Thomas, P., and Lombard, C., "Geometric Conservation Law and Application to Flow Computations on Moving Grids," AIAA Journal, Vol. 10, No. 10, 1979, pp. 1030-1037. doi: $10.2514 / 3.61273$

[22] Kamakoti, R., and Shyy, W., "Evaluation of Geometric Conservation Law Using Pressure-Based Fluid Solver and Moving Grid Technique," International Journal of Heat and Fluid Flow, Vol. 14, No. 7, 2004, pp. $851-865$ doi:10.1108/09615530410546254

[23] Vandenheede, R. B. R., "Force Generation of Bio-Inspired Hover Kinematics: An Experimental Campaign," M.S. Thesis, Delft Univ. of Technology, Delft, The Netherlands, June 2012.

[24] Shyy, W., and Liu, H., "Flapping Wings and Aerodynamic Lift: The Role of Leading-Edge Vortices," AIAA Journal, Vol. 45, No. 12, 2007, pp. 2817-2819. doi: $10.2514 / 1.33205$

[25] Dickinson, M. H., Lehmann, F.-O., and Sane, S. P., "Wing Rotation and the Aerodynamic Basis of Insect Flight," Science, Vol. 284, No. 5422, 1999, pp. 1954-1960. doi: $10.1126 /$ science. 284.5422 .1954

R. Gordnier Associate Editor 